\title{
COVID-19 Outbreak: Current scenario and future prospect in Bangladesh
}

\author{
Afroza Sultana ${ }^{1}$, Otun Saha ${ }^{2}$, and Md. Mizanur Rahaman ${ }^{3}$ \\ ${ }^{1}$ Noakhali Science and Technology University \\ ${ }^{2}$ Affiliation not available \\ ${ }^{3}$ University of Dhaka
}

June 1,2020

COVID-19 Outbreak: Current scenario and Future prospect in Bangladesh Afroza Sultana ${ }^{1 \#}$, Otun Saha ${ }^{2 \#}$, Md. Mizanur Rahaman ${ }^{2 *}$

1Department of Microbiology, Noakhali Science and Technology University, Noakhali 3814, Bangladesh.

2Department of Microbiology, University of Dhaka, Dhaka 1000, Bangladesh

\# Equal Contribution: Jointly Declared as first author due to their equal contribution.

*Correspondence: razu002@du.ac.bd; +8801796585290

Dear Editor,

Coronavirus disease 2019 (COVID-19), which $1^{\text {st }}$ originated in Wuhan, China, and has since spread around the world, a global pandemic. As of May 30, 2020, 6,080,270 cases of COVID-19 have been confirmed in more than 210 countries and territories and at least 368,066 deaths have been reported from around the world (https://www.worldometers.info/coronavirus/). COVID-19 is now establishing a foothold in impoverished, overpopulated and crowded nations, such as Bangladesh. Although the health-care system in Bangladesh has improved over the past few years, its preparedness for a prompt and functional response to the COVID19 outbreak is still questionable as there is still a very poor healthcare management system. ${ }^{1}$ After the first confirmed case of COVID-19 was publicized on $7^{\text {th }}$ March, 2020 although the country was supposedly carrying the virus for quite a longer period according to specialists, Bangladesh may have gone through the most controversial series of events in terms of their taking actions on the matter. The country attempted to bypass the infection rate by promoting self-cleanliness, avoiding physical contact and ordaining health regulations only. However the total number of identified cases so far is 44,608 and total deaths have already crossed 610 . Though the statistics that were published by the authorities has been criticized and failed to achieve faith by many people, the number of tests per million of people is very low which is so far $10 /$ million people with only approximately 10000 tests/day now with a population of 180 million. There has been severe mismanagement of COVID-19 sampling and tests and hence many laboratories already saw contaminations and even though the number of hospitalized patients has not been go high so far, a total of approximately 1000 Doctors and healthcare nurses became infected with SARS-CoV-2. The reasons for this is the poor healthcare management system in Bangladesh with lack of appropriate safety measures including appropriate PPE under the outbreak of such a highly infectious agent.

Controlling the pandemic in Bangladesh will be complicated by a diverse set of problems, including high number of low-income population as well as daily wagers, public health unawareness, capacity of hospitals compared to the vast population, social corruptions and lack of innovations. Among all these problems, 
most important concern is lack of public awareness of COVID-19 and health literacy, exemplified by the vast number of individuals with confirmed COVID-19. Furthermore, few other traditional practices like shaking hands, hugging, community gatherings in mosques, temples, local market, and the paucity of masks and effective handwashing technique will aggravate the crisis. Although like many Muslim majority countries, Muslims have been instructed to pray at home, all of the mosques in Bangladesh remain open. ${ }^{3}$

Moreover, Bangladesh's developing economy and infrastructure relies heavily on garments sectors. Because of this dependency, any government imposed restrictions put in place will not be as effective and will play vital role in viral transmission. Another concerning issue is the shortage of health-care workers. There are only 3 hospital beds per 10,000 people, 1 skilled health professionals per 2000 people, and 1 nurse per 5000 individuals in Bangladesh ${ }^{4}$; physicians are disproportionately distributed across the country, with 18.2 physicians per 10000 people in urban areas and only 1.1 physicians per 10000 in rural areas ${ }^{1}$ though this should be 22.8 per 10000 people according to WHO's Global Health Workforce Alliance (WHO, 2020). ${ }^{5}$ Bangladesh is one of those few countries where complete health care services are dominated by physicians while other professionals like microbiologists, molecular biologists, pharmacists and related experts are rarely considered for the service. With an overall literacy rate of $72.89 \%$ in Bangladesh, community awareness of imperative public health, sanitation, and hygiene practices and more efficient infection prevention strategies must be placed. Another important issue is that, high number of known COVID-19 comorbidity cases exists and Bangladesh is still suffering many communicable and non-communicable diseases like Diabetes, Cardiovascular diseases, Hypertension, Stroke, Tuberculosis, Malaria, and dengue which may play vital role in COVID-19 destruction in Bangladesh. ${ }^{6}$ This year 268 dengue cases have already been reported in Bangladesh, which is already very high as the severe dengue outbreak that started in April, 2019, caused 179 deaths (https://www.newagebd.net/article/103003/2-new-dengue-patients-detected-in-24hrs-dghs). If Dengue cases rises sharply, it will make the situation worse with the rising COVID-19 cases.

Thus the failure to put early restrictions of incoming flights from COVID-19 affected countries and relatively slower response to put country wide lockdowns have put Bangladesh in the present situation when daily cases are consistently increasing. However, the vast number of people in a small land will be at severe risk and without immediate improvement of healthcare facilities with appropriate tests to isolate the infected cases, such risks would grow higher and higher. Thus appropriate measures should be taken immediately and we strongly rebuke government powers to unite in a collaborative effort combining physicians and all related professionals in the healthcare system to address the serious risk posed by COVID-19 to Bangladesh.

\section{Author's contributions}

O.S., A.S carried out the studies (Data collection and data analysis). O.S., A.S drafted the manuscript. M.M.R. developed the hypothesis, supervised the whole work and critically review the drafted manuscript. All authors read and approved the final manuscript.

\section{Acknowledgments}

The authors would like to acknowledge Bangabandhu Science \& Technology Fellowship Trust for supporting Otun Saha with $\mathrm{PhD}$ fellowship.

\section{Conflict of interest}

The authors declare that the research was conducted in the absence of any commercial or financial relationships that could be construed as a potential conflict of interest.

\section{Funding source}

No Funding

\section{References}

1. Ahamed, M. M., Naznin, R. N., Saha, O., \& Rahaman, M. M. Recommendation of fecal specimen for routine molecular detection of SARS-CoV-2 and for COVID-19 discharge criteria. Pathogens and 
global. Health 2020; 1 .

2. Institute of Epidemiology, Disease Control and Research, COVID-19 Status Bangladesh. Available from: https://www.iedcr.gov.bd/ [Last accessed 30 May, 2020].

3. Nishiura, H. et al. Estimation of the asymptomatic ratio of novel coronavirus infections (COVID-19). Int J Infect Dis 2020. doi:10.1016/j.ijid.2020.03.020

4. Ahmed, S. M., Hossain, M. A., RajaChowdhury, A. M., \& Bhuiya, A. U The health workforce crisis in Bangladesh: shortage, inappropriate skill-mix and inequitable distribution. Human resources for health2011; 9(1): 3 .

5. WHO. A universal truth: no health without a workforce. World Health Organisation (WHO) Report. November, 2013. https://www.who. int/workforcealliance/knowledge/resources/ GHWA-a_universal_truth_report.pdf?ua=1 (accessed May 29, 2020).

6. Hossain, Shah Monir. Non-Communicable Diseases (NCDs) in Bangladesh, An overview. Former Director General of Health Services Ministry of Health and Family Welfare Senior Consultant, PPC, MOHFW Senior Advisor, Eminence. Retrieved 9 September 2017. 